\title{
Assessment of Carotid Artery Stenosis and the Use of Statins
}

\author{
Thomas F. Whayne, J $\mathrm{r}^{1}$ \\ ${ }^{1}$ Division of Cardiovascular Medicine, Gill Heart Institute, University of \\ Kentucky, Lexington, Kentucky \\ Address for correspondence Thomas F. Whayne, Jr, MD, PhD, 326 \\ Wethington Building, 900 South Limestone Street, Lexington, KY \\ Int J Angiol 2015;24:173-178. \\ 40536-0200 (e-mail: twhayn0@uky.edu).
}

\author{
Abstract \\ Keywords \\ - atherosclerosis \\ - cardiovascular risk \\ - carotid artery stenosis \\ - coronary heart \\ disease \\ - peripheral arterial \\ disease \\ - peripheral vascular \\ disease \\ - prevention \\ - statins
}

General thinking has previously centered on managing carotid artery stenosis (CAS) by carotid endarterectomy and subsequently, stenting for higher risk patients. However for CAS and other forms of vascular disease, especially when asymptomatic, there is new emphasis on defining underlying mechanisms. Knowledge of these mechanisms can lead to medical treatments that result in possible atherosclerotic plaque stabilization, and even plaque regression, including in the patient with CAS. For now, the key medication class for a medical approach are the statins. Their use is supported by good cardiovascular clinical trial evidence including some directed carotid artery studies, especially with a demonstrated decrease in carotid intima-media thickness. Procedural controversy still exists but the current era in medicine offers significant support for medical management of asymptomatic CAS while techniques to recognize the vulnerable plaque evolve. If CAS converts to a symptomatic status, early referral for endarterectomy or stenting is indicated.
The medical management of vascular disease, especially when directed to avoid surgery or a vascular interventional procedure, continues to evoke controversy and disagreement. However, there is much to support medical management of carotid artery stenosis (CAS) even when asymptomatic and in conjunction with a procedure. Regardless of an asymptomatic, primary, or secondary focus, the use of a statin is key and indicated during this era of aggressive vascular disease management. The multiple issues relevant to this in the patient with CAS have been discussed in this review.

\section{Basic Definitions and Forms of Vascular Disease}

CAS is a component of peripheral vascular disease (PVD) and peripheral arterial disease (PAD). CAS, as a subcategory of PVD, appears to involve a similar atherosclerotic process to that of PAD but the carotid arteries are involved instead. An American Heart Association Symposium in 2008 proposed that PAD should refer specifically to stenosis or occlusion of upper extremity or lower extremity arteries only. Renal, coronary, cerebral, and mesenteric arterial disease as well as all aneurysms are not classified as PAD but are included in the category of PVD. ${ }^{1}$ Specifically, PAD involves the upper and lower extremities although usually the lower extremities are affected. On the other hand, PVD refers to all cardiovascular disease separate from cerebral arterial disease and coronary heart disease (CHD). ${ }^{2}$ Confusion exists because PVD and PAD have been used interchangeably and incorrectly in the past. PAD affects approximately 8 million individuals aged $\geq$ 40 years in the United States. ${ }^{1,3}$

Vascular disease is an all-encompassing term that refers to the entire range of cardiovascular arterial disease. In the case of PVD as a form of vascular disease, the best data regarding prevalence involve PAD. PAD prevalence increases from a $<3 \%$ presence at 50 to 59 years of age to $>25 \%$ of males aged $\geq$ 80 years. ${ }^{3}$ The manifestations of PAD are not always clinically overt. Up to $40 \%$ of PAD individuals experience no symptoms while awareness of existing PAD is approximately 25\%., Although significantly prevalent, PAD remains underdiagnosed and undertreated because of the absence of symptoms and the lack of overt disease manifestations. ${ }^{3-5}$ published online

June 5, 2015
Copyright $\odot 2015$ by Thieme Medical Publishers, Inc., 333 Seventh Avenue, New York, NY 10001, USA. Tel: +1(212) 584-4662.
DOI http://dx.doi.org/ 10.1055/s-0035-1554910. ISSN 1061-1711. 
It is relevant to refer to PAD in a review of CAS since the significance of PAD is not only its involvement of the upper and especially lower extremity arteries but the fact that the presence of PAD indicates a high probability of generalized vascular atherosclerosis. ${ }^{6,7}$ This includes a high prevalence of other arterial diseases, namely CHD, CAS, and abdominal aortic aneurysm (AAA). ${ }^{6,7}$ Also, there is a two-way relationship, in that patients with CHD, CAS, and AAA have a high prevalence of PAD. $^{8-10}$

Over and above the usual stroke and transient ischemic attack (TIA), CAS displays some unique and unusual presentations. A transient postprandial visual loss was reported by Levin and Mootha as an occurrence after eating a meal. ${ }^{11}$ This occurrence was considered to result from decreased perfusion of the choroidal and retinal circulations in association with severe CAS. Cerebrovascular reserve can be defined as the inverse of the potential for cerebral ischemia in the case of severe CAS. In this case, reduction of cerebral perfusion pressure results in autoregulation of the cerebral vasculature with maximal dilation of cerebral arterioles to maintain cerebral flow. ${ }^{12}$ Low cerebrovascular reserve results in increased potential for cerebral ischemia and appears to increase the risk of stroke.

\section{Diagnosis of Carotid Artery Stenosis}

There have been significant advances in the diagnosis of CAS and if implemented widely, diagnosis can be made early. With a serious effort aimed at altering lifestyle and managing risk, the quality of life of patients can be improved and cerebrovascular complications can be reduced. There are many noninvasive tests available for the diagnosis of CAS which are best performed in a vascular laboratory. Standardization and selection of the best imaging test is a significant problem. In an attempt to address this problem, the Canadian Atherosclerosis Imaging Network was established by the Canadian Institutes of Health Research and the Canada Foundation for Innovation to promote significant clinical research, improve the clinical evaluation of vascular disease associated with cardiac and neurologic vascular problems, improve understanding of underlying disease processes, and improve disease prevention approaches. ${ }^{1,13}$

\section{Intra-Arterial Angiography}

The United Kingdom has a program to assess whether imaging modalities, alone or in combination, have the capability to replace intra-arterial (IA) angiography. These imaging modalities include ultrasound (US), magnetic resonance (MR) angiography enhanced with contrast, and computed tomography (CT) angiography. ${ }^{14}$ IA angiography has been the gold standard to evaluate CAS. Assessment of the cost effectiveness of less-invasive imaging tests, the effect on stroke/mortality incidence, and the occurrence and cost of carotid endarterectomies is of major clinical significance. This applies especially if improved access to essential endarterectomy ${ }^{14}$ and other procedures such as angioplasty/stenting result. Carotid angioplasty/stenting has assumed importance as an alternative to carotid endarterectomy for patients at significantly in- creased surgical risk and for a CAS not accessible to a carotid endarterectomy approach. ${ }^{15}$

\section{Duplex Ultrasound}

Duplex US scanning of the arterial system can be specifically applied to CAS. ${ }^{16}$ This technique is one of the most common noninvasive approaches used by a vascular laboratory to define anatomy, hemodynamics, and lesion morphology. This technique utilizes B-mode imaging, pulse wave Doppler, continuous wave Doppler, and color Doppler display. The sensitivity of duplex ultrasonography to detect occlusions and stenoses has been reported to be 95 and $92 \%$, respectively, with specificities of 99 and $97 \%$, respectively. ${ }^{17}$ Some limitations with Doppler imaging include evaluation of tandem stenoses. ${ }^{18}$ An area of stenosis is localized with color Doppler and assessed by measuring Doppler velocities at several arterial sites. The normal arterial velocity waveform is triphasic. It consists of an initial forward flow systolic peak, then a reversal of flow in early diastole, and finally forward flow in late diastole. With progressive arterial disease, there is elimination of reverse flow, a decrease in systolic peak, and an increase in flow in diastole.

\section{Computed Tomography Angiography}

IA angiography has been the gold standard for carotid arterial imaging. It is considered to be the only method that defines the anatomy to an extent required by both surgeons and interventionalists. It is considered the optimal method to help choose the best therapeutic option in vascular patients with its utility validated by years of experience. However, with the recent advances in MR angiography and CT angiography in the last decade, valuable results can be achieved in centers with extensive experience in performing and interpreting these studies. Multidetector CT (MDCT) angiography, while requiring the use of contrast and some exposure to radiation, offers some unique advantages over MR angiography. It can be performed in patients with metallic foreign bodies such as metal stents and pacemakers. In addition, there is a relatively simple protocol with shorter time requirements. The technology of MDCT is rapidly evolving and image quality is continually improving. Nevertheless, severely calcified arteries are difficult to evaluate with CT angiography.

\section{Vascular Assessment with Magnetic Resonance Angiography}

Contrast-enhanced MR angiography using gadolinium as the contrast agent offers excellent images. It was developed to provide another noninvasive alternative to diagnostic CT angiography in the evaluation of vascular disease. In a review and meta-analysis of contrast-enhanced CT angiography, Debrey et al in 2008 reported a sensitivity of $94.6 \%$ (range, 92.4-96.4\%) for detection of severe internal carotid artery stenoses with a specificity of $91.9 \%$ (range, 90.3$93.4 \%){ }^{19}$ MR angiography is noninvasive other than a simple intravenous gadolinium injection. There is no exposure to harmful ionizing radiation to either the patient or the operator. Also, MR angiography offers great detail of the associated surrounding structures (bone, soft tissue). In 
addition, MR angiography can contribute stroke-risk information beyond simple stenosis measurement due to its ability to define specific atherosclerotic plaque fibrous cap characteristics such as thinning, rupture, and hemorrhage. MR angiography can also assess the lipid status of any necrotic core $^{20}$ and image the entire carotid artery wall, ${ }^{21}$ allowing the assessment of aggressive cardiovascular risk management. MR angiography also offers the possible documentation of plaque stabilization and/or regression. However, a limitation of MR angiography imaging is that it cannot be applied in the presence of metal clips or stents since these can cause artifacts that mimic vessel occlusions. In addition, patients with pacemakers, defibrillators, and some cerebral aneurysm clips may not be able to be scanned safely. Also, MR angiography performed with gadolinium has been associated with nephrogenic systemic fibrosis (NSF). This is a rare, but serious, condition that occurs in patients with elevated creatinine levels. The U.S. Food and Drug Administration recently made changes to the drug label for gadolinium-based contrast agents (GBCA) to minimize the risk of NSF associated with the use of GBCA in patients with kidney dysfunction. NSF has not been reported in patients with normal kidney function. Patients at greatest risk for developing NSF after receiving GBCA are those with impaired elimination of the drug, patients with acute kidney injury, and patients with chronic severe kidney disease (with a glomerular filtration rate or GFR $<30 \mathrm{~mL} / \mathrm{min} / 1.73 \mathrm{~m}^{2}$ ). Higher than recommended doses or repeat doses of GBCA also appear to increase the risk for NSF. A meta-analysis of MR angiography compared with catheter angiography demonstrated that the sensitivity and specificity of MR angiography for detection of stenoses greater than $50 \%$ were both in the range of 90 to $100 \%$, with greatest accuracy when gadolinium-enhanced MR angiography was used. ${ }^{22}$ Most current studies report similar results, with an agreement between MR angiography and catheter angiography of 91 to $97 \%$.

\section{Optical Coherence Tomography}

A technique to detect high-risk atherosclerotic plaques in CAS, especially with asymptomatic CAS, is of major interest. Optical coherence tomography (OCT) is invasive as an IA procedure but appears to offer a major possibility to assess potentially vulnerable carotid plaques. ${ }^{23}$ This is another imaging modality that is very relevant to assessment of the results of aggressive medical management. An example is the specific capability to evaluate the benefit of statins in patients with CAS.

\section{Basic Concepts of Carotid Artery Stenosis Management}

There is a well-established overlap of PVD, PAD, and CHD. In a study of ABI as a correlate of PAD in 273 patients with mean age of 71 years, it was found that of 155 patients with a very low $A B I$ of $<0.40,130$ (84\%) had three- or four-vessel CHD. ${ }^{24}$ The medical management of PVD, including CAS, centers on decreasing individual cardiovascular risk factors as much as possible.
Stroke is a form of vascular disease, as is atherosclerotic disease of the aorta, carotid arteries, and lower extremity arteries. The Stroke Prevention by Aggressive Reduction in Cholesterol Levels (SPARCL) trial was a randomized, doubleblind study designed to determine whether atorvastatin $80 \mathrm{mg} / \mathrm{d}$ versus placebo would reduce the risk of fatal or nonfatal stroke. ${ }^{25}$ The patients in SPARCL had no known coronary disease and had experienced a stroke or TIA within the previous 6 months. ${ }^{25}$ During a median follow-up of approximately 5 years, $11.2 \%$ of those receiving atorvastatin and $13.1 \%$ of those receiving placebo reached the primary endpoint of fatal or nonfatal stroke. ${ }^{25}$ This represented a 5-year absolute reduction in risk of $2.2 \%$; adjusted hazard ratio (HR), 0.84 ; 95\% confidence interval (CI), 0.71-0.99; and $p=0.03) .{ }^{25}$ The American Heart Association/American Stroke Association (AHA/ASA) recommendations state that on the basis of the SPARCL trial, administration of statin therapy with intensive lipid-lowering effects is recommended for patients with atherosclerotic ischemic stroke or TIA who do not have known CHD. ${ }^{26}$ These recommendations from SPARCL are intended to reduce the risk of stroke and cardiovascular events. ${ }^{26}$ Therefore, there is good clinical trial evidence to support intensive cardiovascular risk factor management in the patient with CAS.

Elevation or increased levels of high-density lipoproteins (HDL) are generally considered favorable for cardiovascular risk status. However, even though this is well-established from an epidemiologic standpoint, there is increasing information that not all HDL are good. In a community-based cohort study using MR spectroscopy in 930 participants aged 45 to 70 years, Qi et al found that cholesterol-overloaded HDL particles were independently associated with worsening of carotid atherosclerosis. ${ }^{27}$

\section{Statins in the Medical Management of Carotid Artery Stenosis}

Despite the role of statins in favorably modifying cardiovascular risk, the potential value of a healthy lifestyle should never be forgotten. Of relevance are the results from the Prevención con Dieta Mediterránea (PREDIMED) trial ${ }^{28}$ which showed that consuming a Mediterranean diet with the addition of nuts (in this case, extra virgin olive oil as a supplement did not make a difference) delayed the increase in carotid intima-media thickness (CIMT) and plaque progression. ${ }^{28}$ Similar results were seen with an intensive multiple cardiovascular risk factor intervention in 97 patients with type 2 diabetes mellitus (DM). Over 2 years, an intensive risk factor intervention resulted in a significant decrease in CIMT. $^{29}$ There is ample evidence that statins appear to decrease the progression of CIMT, may improve peripheral endothelial function, and offer the possibility of reversing atherosclerosis. $^{30}$ The Stop Atherosclerosis in Native Diabetics Study (SANDS) found that in patients with type 2 $\mathrm{DM}$, reducing both low-density lipoprotein cholesterol (LDL-C) to an aggressive target of $70 \mathrm{mg} / \mathrm{dL}$ and systolic blood pressure to $115 \mathrm{~mm} \mathrm{Hg}$ resulted in regression of CIMT and a decrease in left ventricular mass. ${ }^{31}$ In SANDS, a statin was 
started if lifestyle modification failed to attain the desired LDL goal; if this did not occur with the addition of a statin, ezetimibe was added.

LDL-C is the most specific lipoprotein target associated with $\mathrm{CHD},{ }^{32}$ and as such is considered the gold standard of CHD risk factors. LDL-C is the only lipoprotein with strong evidence to support its reduction as beneficial in decreasing acute and chronic cardiovascular events ${ }^{33}$ and associated diseases including PAD. Of all the medications available to manage and decrease LDL-C, statins are the cornerstone and the first medication to initiate after restricting cholesterol and saturated fat intake. For the high-risk cardiovascular patient, including any patient with proven CHD, PAD, DM, or multiple cardiovascular risk factors, the goal for LDL-C is now established as $<70 \mathrm{mg} / \mathrm{dL}^{34}$

Physicians and surgeons caring for patients with CHD/ $\mathrm{PAD} / \mathrm{DM} /$ metabolic syndrome (MS) need to be aware of the intertwined associated cardiovascular risk factors to optimize the treatment. Although the lowering of LDL-C to below $70 \mathrm{mg} / \mathrm{dL}$ in the DM patient who has established high cardiovascular risk is indicated, close plasma glucose control of DM has only a minimal association with decreased cardiovascular risk. Decreased cardiovascular risk as related to glucose control was minimal in the Action in Diabetes and Vascular Disease Preterax and Diamicron Modified Release Controlled Evaluation (ADVANCE) trial. ${ }^{35}$ In contrast, close control of glucose has been associated with problems as shown in the Action to Control Cardiovascular Risk in Diabetes (ACCORD) trial. ${ }^{36}$ In the case of MS, all components should be treated aggressively as part of obligatory preventive management. The controversy over the definitions and etiology of MS as a clinical aggregate or collection of abnormalities in a population with bad health habits will continue. ${ }^{37-39}$ Nevertheless, efforts are underway to elucidate and understand the relationships. Maximum treatment of each component has support such as the increase in CIMT related to each MS component added. ${ }^{40}$

The Pravastatin or Atorvastatin Evaluation and Infection Therapy-Thrombolysis In Myocardial Infarction 22 (PROVE ITTIMI 22) substudy showed that greatest clinical benefit was achieved in acute coronary syndrome patients who reached an LDL-C goal of $<40 \mathrm{mg} / \mathrm{dL}$ or 40 to $60 \mathrm{mg} / \mathrm{dl}^{41}{ }^{41}$ Therefore, "lower is better" for LDL-C appears to be well-established for CHD with no proven downside. On the other hand, the Ezetimibe and Simvastatin in Hypercholesterolemia Enhances Atherosclerosis Regression (ENHANCE) trial studied the effect of ezetimibe plus simvastatin versus simvastatin alone on CIMT with controversial results. ENHANCE showed no significant difference in CIMT between the two groups and no significant difference in cardiovascular outcomes. However, the study was a short-term, small, imaging study utilizing the highest risk group of cardiovascular patients consisting of heterozygous familial hypercholesterolemia patients. This is a situation where it may be most difficult to show a difference, especially over a short time period. ${ }^{42}$ Thus far, it can be considered that the study failed to negate the importance of maximum LDL-C lowering in the high-risk cardiovascular patient. Ezetimibe contributes significantly by contributing up to an additional 24\% LDL-C reduction while keeping statins at a safer, lower, long-term dose. ${ }^{43}$ This occurs while still preserving the majority of statin LDL-C reduction and beneficial pleiotropic effects. ${ }^{43}$

Despite excellent evidence supporting the beneficial effects of statins in cardiovascular risk management, the specific benefit of statins has been more problematic in the case of PAD and CAS patients, particularly perioperative outcomes. Nevertheless, all vascular patients should be on a statin for secondary cardiovascular disease prevention. ${ }^{44}$ In a study of 328 patients with asymptomatic CAS scheduled for elective carotid endarterectomy, Heyer et al reported that preoperative statin use was associated with decreased neurological injury following endarterectomy ${ }^{45}$ Such data, of course, eliminates the possibility of a prospective randomized trial. Furthermore, age is not a qualifying issue and elderly patients with a history of stroke or TIA are benefited by the use of a statin. ${ }^{46}$

Significant but asymptomatic CAS still evokes controversy regarding an invasive endarterectomy or stenting approach, since this is being done in approximately $70 \%$ of the asymptomatic cases. Nevertheless, there is increasing evidence to consider asymptomatic CAS a medical condition. ${ }^{47}$ Unless microemboli are identified on transcranial Doppler, the 20 year risk of stroke in asymptomatic CAS is $1 \%$ or less. ${ }^{47}$ Unfortunately, there are surgical and interventional conflicts involved regarding asymptomatic CAS. Billed as a transatlantic debate, Schneider and Naylor recognized the need for an adequately powered trial with treatment arms for endarterectomy, stenting, and best medical therapy. In contrast, Naylor concluded that a very large majority of asymptomatic CAS patients will never develop a stroke, only $1 \%$ of strokes will be prevented by a mass campaign of intervention, and $94 \%$ of interventions in asymptomatic CAS patients may be unnecessary. ${ }^{48}$ It can be effectively argued that only where carotid endarterectomy can be performed with a combined perioperative stroke and death rate of $<3 \%$ should endarterectomy be advised for asymptomatic CAS, prompting some physicians to avoid a routine screening policy. ${ }^{49}$ Therefore, a specific correct approach to asymptomatic CAS has not been established but nevertheless, conversion to symptomatic status should lead to urgent medical attention. In the future, procedures such as OCT may identify vulnerable carotid plaques that should be urgently managed with an invasive approach.

\section{Prognosis of Carotid Artery Stenosis}

The purpose of intensive medical management for any vascular disease, including CAS, is in small part to aid symptom relief. Nevertheless, prevention is the major goal with a desired result of improving prognosis. Van Kuijk et al screened 2,933 consecutive patients with symptomatic PAD before surgery for their PAD. ${ }^{50}$ The screening was done for associated cerebrovascular disease and $\mathrm{CHD}$, bringing the assessment to a total of three affected vascular beds (AVB). During a median follow-up of 6 years, 1,398 patients died with $54 \%$ of the deaths secondary to a cardiovascular etiology. 
After adjustment for baseline risk factors and discharge medications, a HR with $\mathrm{CI}$ was determined and the presence of two and three AVBs was associated with increased allcause mortality (HR 1.3, 95\% CI 1.2-1.5; HR 1.8, 95\% CI 1.52.2 , respectively) and increased cardiovascular mortality (HR 1.5, 95\% CI 1.2-1.7; HR 2.0, 95\% CI 1.6-2.5, respectively). The results showed that involvement of multiple vascular beds in PAD patients is a significant and independent risk factor for all-cause and cardiovascular mortality.

On the other hand, Welten et al reported that the longterm prognosis of PVD surgery patients is significantly worse than for CHD patients. ${ }^{51}$ They studied 2,730 patients undergoing PVD surgery and placed them into four groups as follows: carotid endarterectomy for CAS $(n=560)$, elective abdominal aortic aneurysm (AAA) surgery $(n=923)$, acute AAA surgery $(n=200)$, and lower limb reconstruction procedures $(n=1,047)$. The lower limb procedures consisted of iliac-femoral, femoral-popliteal, or femoral-tibial artery bypass; removal of infected prostheses; opening of peripheral obstructions; and other elective peripheral arterial surgical reconstructions. The PVD patients had a poorer long-term prognosis (HR 2.40, 95\% CI 2.18-2.65) than CHD patients $(p<0.001)$. The major cause of death in these PVD patients was cerebrovascular complications (46\%). There was no significant difference in long-term survival noted between AAA and lower limb reconstruction groups ( $\log r$ and $p=0.70)$.

\section{Conclusions}

An aggressive medical cardiovascular risk reduction approach, centered around the use of a statin when CAS is present, is the current standard of care, supported by ample clinical trial evidence. Therefore, it can be unequivocally stated that any patient with CAS should be aggressively managed with a statin to achieve LDL-C reduction to less than $70 \mathrm{mg} / \mathrm{dL}$, $^{52}$ or an even lower LDL-C level if the initial one was close to $70 \mathrm{mg} / \mathrm{dL}$. This can be recommended despite failure to obtain agreement on the use of endarterectomy, stenting, or medical management only, regardless of whether or not the CAS is asymptomatic or symptomatic.

\section{Conflicts of Interest}

The author has no conflict of interest to declare involving any pharmaceutical company, medical device company, stock ownership, or any other possible perceived conflict.

\section{Acknowledgment}

The author wishes to recognize the excellent editorial critique and contribution of Paula Heron to this article.

\section{References}

1 Hiatt WR, Goldstone J, Smith SC Jr, et al; American Heart Association Writing Group 1. Atherosclerotic Peripheral Vascular Disease Symposium II: nomenclature for vascular diseases. Circulation 2008;118(25):2826-2829
2 American Heart Association. What Is Peripheral Vascular Disease? Available at: http://www.heart.org/idc/groups/heart-public/ $@$ @cm/@hcm/documents/downloadable/ucm_300323.pdf. Accessed January 30, 2015

3 Rooke TW, Hirsch AT, Misra S, et al; American College of Cardiology Foundation/American Heart Association Task Force on Practice Guidelines; Society for Cardiovascular Angiography and Interventions; Society of Interventional Radiology; Society for Vascular Medicine; Society for Vascular Surgery. 2011 ACCF/AHA focused update of the guideline for the management of patients with peripheral artery disease (updating the 2005 guideline): a report of the American College of Cardiology Foundation/American Heart Association Task Force on Practice Guidelines: developed in collaboration with the Society for Cardiovascular Angiography and Interventions, Society of Interventional Radiology, Society for Vascular Medicine, and Society for Vascular Surgery. J Vasc Surg 2011;54(5):e32-e58

4 Stansby G, Mister R, Fowkes G, et al; Prospective Registry and Evaluation of Peripheral Arterial Risks, Events and Distribution Investigators. High risk of peripheral arterial disease in the United Kingdom: 2-year results of a prospective registry. Angiology 2011; 62(2):111-118

5 Mukherjee D, Lingam P, Chetcuti S, et al. Missed opportunities to treat atherosclerosis in patients undergoing peripheral vascular interventions: insights from the University of Michigan Peripheral Vascular Disease Quality Improvement Initiative (PVD-QI2). Circulation 2002;106(15):1909-1912

6 Shah AM, Banerjee T, Mukherjee D. Coronary, peripheral and cerebrovascular disease: a complex relationship. J Indian Med Assoc 2010;108(5):292-294, 296

7 Mukherjee D, Cho L. Peripheral arterial disease: considerations in risks, diagnosis, and treatment. J Natl Med Assoc 2009;101(10): 999-1008

8 Takigawa M, Yoshimuta T, Akutsu K, Takeshita S, Yokoyama N. Prevalence and predictors of coexistent silent atherosclerotic cardiovascular disease in patients with abdominal aortic aneurysm without previous symptomatic cardiovascular diseases. Angiology 2012;63(5):380-385

9 Paraskevas KI, Mikhailidis DP, Liapis CD. Internal carotid artery occlusion: association with atherosclerotic disease in other arterial beds and vascular risk factors. Angiology 2007;58(3):329-335

10 Badheka AO, Rathod AD, Bharadwaj AS, et al. Outcomes and risk prediction model for peripheral arterial disease in patients with stable coronary artery disease. Angiology 2011;62(6):473-479

11 Levin LA, Mootha VV. Postprandial transient visual loss. A symptom of critical carotid stenosis. Ophthalmology 1997;104(3): 397-401

12 Gupta A, Chazen JL, Hartman M, et al. Cerebrovascular reserve and stroke risk in patients with carotid stenosis or occlusion: a systematic review and meta-analysis. Stroke 2012;43(11): 2884-2891

13 Tardif JC, Spence JD, Heinonen TM, et al. Atherosclerosis imaging and the Canadian Atherosclerosis Imaging Network. Can J Cardiol 2013;29(3):297-303

14 Wardlaw JM, Chappell FM, Stevenson M, et al. Accurate, practical and cost-effective assessment of carotid stenosis in the UK. Health Technol Assess 2006;10(30):iii-iv, ix-X, 1-182

15 Chaer RA, DeRubertis B, Patel S, Lin SC, Kent CK, Faries PL. Current management of extracranial carotid artery disease. Rev Recent Clin Trials 2006;1(3):293-301

16 Kohler TR, Nance DR, Cramer MM, Vandenburghe N, Strandness DE Jr. Duplex scanning for diagnosis of aortoiliac and femoropopliteal disease: a prospective study. Circulation 1987;76(5): 1074-1080

17 Whelan JF, Barry MH, Moir JD. Color flow Doppler ultrasonography: comparison with peripheral arteriography for the investigation of peripheral vascular disease. J Clin Ultrasound 1992;20(6): 369-374 
18 Allard L, Cloutier G, Durand LG, Roederer GO, Langlois YE. Limitations of ultrasonic duplex scanning for diagnosing lower limb arterial stenoses in the presence of adjacent segment disease. J Vasc Surg 1994;19(4):650-657

19 Debrey SM, Yu H, Lynch JK, et al. Diagnostic accuracy of magnetic resonance angiography for internal carotid artery disease: a systematic review and meta-analysis. Stroke 2008;39(8): 2237-2248

20 Gupta A, Baradaran H, Schweitzer AD, et al. Carotid plaque MRI and stroke risk: a systematic review and meta-analysis. Stroke 2013;44(11):3071-3077

21 Zhang Y, Guallar E, Qiao Y, Wasserman BA. Is carotid intima-media thickness as predictive as other noninvasive techniques for the detection of coronary artery disease? Arterioscler Thromb Vasc Biol 2014;34(7):1341-1345

22 Nelemans PJ, Leiner T, de Vet HC, van Engelshoven JM. Peripheral arterial disease: meta-analysis of the diagnostic performance of MR angiography. Radiology 2000;217(1):105-114

23 Cilingiroglu M, Hakeem A, Feldman M, Wholey M. Optical coherence tomography imaging in asymptomatic patients with carotid artery stenosis. Cardiovasc Revasc Med 2013;14(1):53-56

24 Sukhija R, Aronow WS, Yalamanchili K, Peterson SJ, Frishman WH, Babu S. Association of ankle-brachial index with severity of angiographic coronary artery disease in patients with peripheral arterial disease and coronary artery disease. Cardiology 2005; 103(3):158-160

25 Amarenco P, Bogousslavsky J, Callahan A III, et al; Stroke Prevention by Aggressive Reduction in Cholesterol Levels (SPARCL) Investigators. High-dose atorvastatin after stroke or transient ischemic attack. N Engl J Med 2006;355(6):549-559

26 Adams RJ, Albers G, Alberts MJ, et al; American Heart Association; American Stroke Association. Update to the AHA/ASA recommendations for the prevention of stroke in patients with stroke and transient ischemic attack. Stroke 2008;39(5):1647-1652

27 Qi Y, Fan J, Liu J, et al. Cholesterol-overloaded HDL particles are independently associated with progression of carotid atherosclerosis in a cardiovascular disease-free population: a communitybased cohort study. J Am Coll Cardiol 2015;65(4):355-363

28 Sala-Vila A, Romero-Mamani ES, Gilabert R, et al. Changes in ultrasound-assessed carotid intima-media thickness and plaque with a Mediterranean diet: a substudy of the PREDIMED trial. Arterioscler Thromb Vasc Biol 2014;34(2):439-445

29 Tripolt NJ, Narath SH, Eder M, Pieber TR, Wascher TC, Sourij H. Multiple risk factor intervention reduces carotid atherosclerosis in patients with type 2 diabetes. Cardiovasc Diabetol 2014;13:95

30 Balk EM, Karas RH, Jordan HS, Kupelnick B, Chew P, Lau J. Effects of statins on vascular structure and function: a systematic review. Am J Med 2004;117(10):775-790

31 Howard BV, Roman MJ, Devereux RB, et al. Effect of lower targets for blood pressure and LDL cholesterol on atherosclerosis in diabetes: the SANDS randomized trial. JAMA 2008;299(14): 1678-1689

32 Sytkowski PA, Kannel WB, D’Agostino RB. Changes in risk factors and the decline in mortality from cardiovascular disease. The Framingham Heart Study. N Engl J Med 1990;322(23):1635-1641

33 Whayne TF. Evaluation critique of state of the art dyslipidemia management in general and with a special emphasis on the Indian population. Indian Heart J 2007;59(3):218-225

34 Ballantyne CM. Cholesterol, lipids, and statins. Tex Heart Inst J 2005;32(3):378-379

35 Patel A, MacMahon S, Chalmers J, et al; ADVANCE Collaborative Group. Intensive blood glucose control and vascular outcomes in patients with type 2 diabetes. N Engl J Med 2008;358(24): 2560-2572

36 Gerstein HC, Miller ME, Byington RP, et al; Action to Control Cardiovascular Risk in Diabetes Study Group. Effects of intensive glucose lowering in type 2 diabetes. N Engl J Med 2008;358(24): 2545-2559

37 Takamiya T, Zaky WR, Edmundowics D, et al. World Health Organization-defined metabolic syndrome is a better predictor of coronary calcium than the adult treatment panel III criteria in American men aged 40-49 years. Diabetes Care 2004;27(12): 2977-2979

38 Balkau B, Charles MA; European Group for the Study of Insulin Resistance (EGIR). Comment on the provisional report from the WHO consultation. Diabet Med 1999;16(5):442-443

39 A new worldwide definition of the metabolic syndrome: Consensus from the International Diabetes Federation could help stop the cardiovascular disease time bomb [press release]. Berlin, German: International Diabetes Federation; April 14, 2005

40 Adolphe A, Cook LS, Huang X. A cross-sectional study of intimamedia thickness, ethnicity, metabolic syndrome, and cardiovascular risk in 2268 study participants. Mayo Clin Proc 2009;84(3): 221-228

41 Wiviott SD, Cannon CP, Morrow DA, Ray KK, Pfeffer MA, Braunwald E; PROVE IT-TIMI 22 Investigators. Can low-density lipoprotein be too low? The safety and efficacy of achieving very low low-density lipoprotein with intensive statin therapy: a PROVE IT-TIMI 22 substudy. J Am Coll Cardiol 2005;46(8):1411-1416

42 Whayne TF Jr. Is there a problem with ezetimibe or just ENHANCEd hype? Angiology 2008;59(6):661-663

43 Mikhailidis DP, Sibbring GC, Ballantyne CM, Davies GM, Catapano AL. Meta-analysis of the cholesterol-lowering effect of ezetimibe added to ongoing statin therapy. Curr Med Res Opin 2007;23(8): 2009-2026

44 Stalenhoef AF. The benefit of statins in non-cardiac vascular surgery patients. J Vasc Surg 2009;49(1):260-265

45 Heyer EJ, Mergeche JL, Bruce SS, et al. Statins reduce neurologic injury in asymptomatic carotid endarterectomy patients. Stroke 2013;44(4):1150-1152

46 Alhusban A, Fagan SC. Secondary prevention of stroke in the elderly: a review of the evidence. Am J Geriatr Pharmacother 2011;9(3):143-152

47 Spence JD. Asymptomatic carotid stenosis: mainly a medical condition. Vascular 2010;18(3):123-126, discussion 127-129

48 Schneider PA, Naylor AR. Transatlantic debate. Asymptomatic carotid artery stenosis-medical therapy alone versus medical therapy plus carotid endarterectomy or stenting. Eur J Vasc Endovasc Surg 2010;40(2):274-281

49 Ederle J, Brown MM. The evidence for medicine versus surgery for carotid stenosis. Eur J Radiol 2006;60(1):3-7

50 van Kuijk JP, Flu WJ, Welten GM, et al. Long-term prognosis of patients with peripheral arterial disease with or without polyvascular atherosclerotic disease. Eur Heart J 2010;31(8): 992-999

51 Welten GM, Schouten O, Hoeks SE, et al. Long-term prognosis of patients with peripheral arterial disease: a comparison in patients with coronary artery disease. J Am Coll Cardiol 2008;51(16): 1588-1596

52 Grundy SM, Cleeman JI, Merz CN, et al; National Heart, Lung, and Blood Institute; American College of Cardiology Foundation; American Heart Association. Implications of recent clinical trials for the National Cholesterol Education Program Adult Treatment Panel III guidelines. Circulation 2004;110(2):227-239 